\title{
Significance tests for the wavelet power and the wavelet power spectrum
}

\section{Z. Ge}

Research associate of the National Research Council; Ecosystems Research Division, NERL, USEPA, 960 College Station Road, Athens, Georgia 30605, USA

Received: 28 June 2007 - Revised: 18 October 2007 - Accepted: 21 November 2007 - Published: 29 November 2007

\begin{abstract}
Significance tests usually address the issue how to distinguish statistically significant results from those due to pure randomness when only one sample of the population is studied. This issue is also important when the results obtained using the wavelet analysis are to be interpreted. Torrence and Compo (1998) is one of the earliest works that has systematically discussed this problem. Their results, however, were based on Monte Carlo simulations, and hence, failed to unveil many interesting and important properties of the wavelet analysis. In the present work, the sampling distributions of the wavelet power and power spectrum of a Gaussian White Noise (GWN) were derived in a rigorous statistical framework, through which the significance tests for these two fundamental quantities in the wavelet analysis were established. It was found that the results given by Torrence and Compo (1998) are numerically accurate when adjusted by a factor of the sampling period, while some of their statements require reassessment. More importantly, the sampling distribution of the wavelet power spectrum of a GWN was found to be highly dependent on the local covariance structure of the wavelets, a fact that makes the significance levels intimately related to the specific wavelet family. In addition to simulated signals, the significance tests developed in this work were demonstrated on an actual wave elevation time series observed from a buoy on Lake Michigan. In this simple application in geophysics, we showed how proper significance tests helped to sort out physically meaningful peaks from those created by random noise. The derivations in the present work can be readily extended to other wavelet-based quantities or analyses using other wavelet families.
\end{abstract}

Keywords. Meteorology and atmospheric dynamics (Instruments and techniques) - Oceanography: physical (Surface waves and tides) - General or miscellaneous (Techniques applicable in three of more fields)

Correspondence to: $\mathrm{Z}$. Ge

(ge.zhongfu@epa.gov)

\section{Introduction}

After two decades of fast development, the wavelet analysis has become a powerful and effective tool for analysing nonlinear and especially nonstationary time series in many disciplines (Addison, 2002). In particular, its application in geophysics has made great contribution to the advancement of the theory and the practice of the wavelet analysis (e.g. Goupilland et al., 1984; Foufoula-Georgiou and $\mathrm{Ku}-$ mar, 1994). To date, the implementation of the wavelet analysis has become easy for many researchers, owing to the work of Torrence and Compo (1998) (referred to TC98 hereafter). In this widely-acknowledged paper, which has been cited over 1000 times as of June of 2007 (ISI Web of knowledge), many practical issues for the wavelet analysis are discussed, accompanied by source codes in Fortran and Matlab posted on their website (http://atoc.colorado.edu/ research/wavelets/). Moreover, TC98 is one of the earliest works that gives a guide for conducting significance tests for the wavelet power, auto-, and cross-spectrum, which is pertinent to the present work.

The significance test for the wavelet analysis is undoubtedly important due to the simple fact that there always seem to be some patterns (e.g. peaks) in the wavelet scalogram even if the analysed signal is pure noise. In this case, a bottom line must be drawn below which no conclusion can be made based on the results. This issue should receive especial attention when the continuous wavelet transform (CWT) is employed, because the CWT may introduce considerable redundancy in the results (Mallat, 1998, p. 79). However, the significance levels established in TC98 were based on a series of Monte Carlo simulations instead of reasoning in a rigorous statistical framework. Although simple and straightforward, their approach failed to disclose many interesting and essential properties that could have been shown through an analytical approach. This paper aims to pick up what they have left behind, and, furthermore, to show the necessity and effectiveness of proper significance tests. Significance

Published by Copernicus Publications on behalf of the European Geosciences Union. 
levels for the wavelet power and the wavelet power spectrum are proposed in the following sections of the present paper. Besides the simulated noisy sine signals, actually observed wave elevation on Lake Michigan of the United States was used as a demonstration for the proposed significance tests. Although, for brevity, only CWT using the Morlet wavelet is considered in the present paper, the methodologies described here can be readily extended to the discrete wavelet transform and the wavelet analysis based on other wavelet families, such as the Mexican hat and the derivative of a Gaussian (DOG) wavelets.

\section{Significance tests developed by TC98}

To aid those readers who are not familiar with TC98, we give a brief introduction of their theories that are relevant to the present work.

The Morlet (mother) wavelet is typically defined as

$\psi(t)=\pi^{-1 / 4} e^{i \omega_{0} t} e^{-\frac{t^{2}}{2}}$,

where $\omega_{0}$ is set equal to 6.0 to approximately satisfy the admissibility condition, and $t$ is used as a more self-explanatory notation for time than $\eta$ in TC98. Then the family of the Morlet wavelets $\psi_{a, b}(t)$ can be generated by replacing the time variable in Eq. (1) with $(t-b) / a$, where $b$ denotes the shift in time and $a$ is the scale variable:

$\psi_{a, b}(t)=\frac{1}{\sqrt{a}} \psi\left(\frac{t-b}{a}\right)$.

The factor $1 / \sqrt{a}$ is used to assure equal energy for wavelets at different scales (Addison, 2002, p. 65).

The wavelet coefficient, $T(a, b)$, obtained from the wavelet transform of a real function $x(t)$ is usually defined as

$T(a, b)=\int_{-\infty}^{\infty} x(t) \psi_{a, b}^{*}(t) d t$

(e.g. Addison, 2002, p. 13). Here $(\cdots)^{*}$ represents the complex conjugate of a given quantity. Hereafter, all integrals will be abbreviated as $\int$ if the integral limits are from $-\infty$ to $\infty$. In a discrete form, TC98 gives an alternative expression for the wavelet coefficient at time index $n$ and scale $a$ :

$W_{n}(a)=\sum_{n^{\prime}=0}^{N-1} x_{n^{\prime}} \psi^{*}\left[\frac{\left(n^{\prime}-n\right) \delta t}{a}\right]$,

where $x_{n}$ is the sampled series of $x(t), N$ denotes the length of the studied time series, and $\delta t$ denotes the sampling period. Nevertheless, this expression is inconsistent with the standard form such as Eq. (3). We found that TC98's wavelet coefficient, $W_{n}(a)$, should be multiplied with square-root of the sampling period, $\delta t$, to be equal to the standardly defined $T(a, b)$, or

$|T(a, b)|^{2}=\delta t\left|W_{n}(a)\right|^{2}$.
More explanations are given in Appendix A. This inconsistency actually stems from the different factor TC98 used to normalize the wavelets for a constant energy over all scales. Specifically, the normalization factor TC98 used is $\sqrt{\delta t / a}$ while in the standard form, Eq. (2), the corresponding term is $1 / \sqrt{a}$. Although TC98's normalization of wavelets always preserves the dimension of $\psi_{a, b}(t)$ to be the same as that of the mother wavelet and hence $W_{n}(a)$ and $x(t)$ will have the same dimension for nondimensional wavelets (Eq. 4), most works in the literature prefer the standard form, Eq. (2), probably because of its mathematical elegance. Compared with Eq. (2), the inclusion of $\delta t$ in a general formula of the wavelet analysis makes the formula dependent on the particular sampling procedure. We will adopt the standard form, Eq. (2), in the remainder of the paper, yet keeping in mind that $T(a, b)$ and $W_{n}(a)$ do have different dimensions, which however does not affect the theories or numerical results of the wavelet analysis.

Based on Monte Carlo simulations, they found that the wavelet power (the squared modulus of the wavelet coefficient, referred to as the local wavelet power spectrum in TC98) of a noise signal normalized by the signal variance in the time domain, $\sigma^{2}$, has a $\chi_{2}^{2}$ distribution:

$\frac{\left|W_{n}(a)\right|^{2}}{\sigma^{2}} \Rightarrow \frac{1}{2} P_{k} \chi_{2}^{2}$,

where $P_{k}$ is the mean spectrum of the background noise at the Fourier frequency $k$, and the symbol $\Rightarrow$ means "is distributed as". In the case of significance tests for waveletbased quantities against a Gaussian White Noise (GWN), $P_{k}$ is identically 1 for all frequencies (or scales). Hereafter, the factor $P_{k}$ will not be included in equations.

The global wavelet spectrum, which is defined as the time average over a series of wavelet powers, can be expressed as

$\bar{W}^{2}(a)=\frac{1}{N} \sum_{n=0}^{N-1}\left|W_{n}(a)\right|^{2}$,

where $N$ is the number of the data points in the time domain that are involved in the wavelet spectrum. Again, based on the Monte Carlo simulations, the 5\% significance level of the normalized (by $\sigma^{2}$ ) global wavelet spectrum of a GWN can be empirically fitted by a chi-square curve, $\chi_{v}^{2} / v$, where the degree-of-freedom $v$ can be numerically estimated through the relation

$v=2 \sqrt{1+\left(\frac{n_{a} \delta t}{\gamma a}\right)^{2}}$

(see TC98 for more details). It is important to note that although the 5\% significance level conforms with a chi-square trend it is difficult to state that the sampling distribution of the wavelet spectrum of a GWN is really a $\chi_{v}^{2} / \nu$. It also is unclear whether the empirical relations for the $5 \%$ significance level such as Eq. (8) can be extended for other significance levels or other integration ranges. Obviously, such deficiency stems from the lack of rigorous statistical reasoning. 


\section{Significance tests for the wavelet scalogram and power spectrum}

As TC98 points out, a significance test should have a specific background distribution presumed. In the present work, we use a GWN process to establish the null hypothesis, so that the significance test determines on what basis one should reject the null hypothesis and infer that the obtained wavelet-based results are not due to white noise. In the cases where the null hypothesis is established based on a red noise, the analysis here can be readily adapted. In the following discussions, the GWN is simply expressed as $x(t)$ which has a zero mean $(E[x(t)]=0)$ and its autocovariance can be expressed with a Kronecker delta function, $\operatorname{Cov}\left[x(t), x\left(t^{\prime}\right)\right]=\delta\left(t-t^{\prime}\right) \sigma^{2}$ (e.g. Jenkins and Watts, 1968, p. 225).

3.1 The sampling distribution of the wavelet power of a GWN

The wavelet power $|T|^{2}$ (the scale and time variables $a$ and $b$ have been omitted for simplicity) equals $\operatorname{Re}^{2}[T]+\operatorname{Im}^{2}[T]$, where $\operatorname{Re}[\cdots]$ and $\operatorname{Im}[\cdots]$ denote the real and imaginary parts, respectively, of a complex number. Using Eq. (3), we have

$\operatorname{Re}[T]=\int x(t) \operatorname{Re}\left[\psi_{a, b}^{*}(t)\right] d t$

and

$\operatorname{Im}[T]=\int x(t) \operatorname{Im}\left[\psi_{a, b}^{*}(t)\right] d t$.

It is evident that both $\operatorname{Re}[T]$ and $\operatorname{Im}[T]$ are normally distributed due to the Gaussianity of $x(t)$.

Noting that the wavelets are all deterministic and hence can be moved out of the operators for random variables such as the expectation and the covariance, we simply have $E[\operatorname{Re}[T]]=E[\operatorname{Im}[T]]=0$ and

$\operatorname{Cov}[\operatorname{Re}[T], \operatorname{Im}[T]]=\operatorname{Cov}\left[\int x(t) \operatorname{Re}\left[\psi_{a, b}^{*}(t)\right] d t\right.$,

$\left.\int x(t) \operatorname{Im}\left[\psi_{a, b}^{*}(t)\right] d t\right]$

$=\iint \operatorname{Cov}\left[x(t), x\left(t^{\prime}\right)\right] \operatorname{Re}\left[\psi_{a, b}^{*}(t)\right] \operatorname{Im}\left[\psi_{a, b}^{*}\left(t^{\prime}\right)\right] d t d t^{\prime}=0($

for $t \neq t^{\prime}$. Furthermore, it can be verified that $\operatorname{Cov}[\operatorname{Re}[T], \operatorname{Im}[T]] \equiv 0$ for $\psi_{a, b}(t)$ being the Morlet wavelets even if $t=t^{\prime}$. Because of the zero-mean property,

$\operatorname{Var}[\operatorname{Re}[T]]=E\left[\operatorname{Re}^{2}[T]\right]=$

$\iint E\left[x(t) x\left(t^{\prime}\right)\right] \operatorname{Re}\left[\psi_{a, b}^{*}(t)\right] \operatorname{Re}\left[\psi_{a, b}^{*}\left(t^{\prime}\right)\right] d t d t^{\prime}$.

Based on the results in Appendix B, it follows that

$\operatorname{Var}[\operatorname{Re}[T]]=\delta t \sigma^{2} \int \operatorname{Re}^{2}\left[\psi_{a, b}^{*}(t)\right] d t$ and

$\operatorname{Var}[\operatorname{Im}[T]]=\delta t \sigma^{2} \int \operatorname{Im}^{2}\left[\psi_{a, b}^{*}(t)\right] d t$.

Equations (11)-(14) implies that at a certain point in the time-scale domain, $(a, b)$, the real and imaginary parts of the wavelet coefficient of a GWN are both zero-mean random variables and are independent of each other. In particular, we can show that, for the Morlet wavelet,

$\int \operatorname{Re}^{2}\left[\psi_{a, b}^{*}(t)\right] d t=\int \operatorname{Im}^{2}\left[\psi_{a, b}^{*}(t)\right] d t=\frac{1}{2}$,

and hence Eqs. (13) and (14) become

$\operatorname{Var}[\operatorname{Re}[T]]=\operatorname{Var}[\operatorname{Im}[T]]=\frac{1}{2} \delta t \sigma^{2}$.

Therefore, only for the Morlet wavelet,

$\frac{|T|^{2}}{\delta t \sigma^{2} / 2}=\frac{\operatorname{Re}^{2}[T]}{\operatorname{Var}[\operatorname{Re}[T]]}+\frac{\operatorname{Im}^{2}[T]}{\operatorname{Var}[\operatorname{Im}[T]]} \Rightarrow \chi_{2}^{2}$,

which leads to

$|T|^{2} \Rightarrow \frac{1}{2} \delta t \sigma^{2} \chi_{2}^{2}$.

Consequently, the wavelet power has a $\chi_{2}^{2}$ distribution only when the wavelet family used is the Morlet wavelet to satisfy the equal-variance condition in Eq. (16). For other wavelet families, the relation (18) might approximately hold but not rigorously. This property was overlooked by TC 98 . Using Eq. (5) for adjustment we obtain $\left|W_{n}(a)\right|^{2} / \sigma^{2} \Rightarrow \frac{1}{2} \chi_{2}^{2}$, in agreement with TC98's result, Eq. (6). The significance test can be conducted as follows. If the normalized wavelet power, $|T|^{2} / \sigma^{2}$, is less than the value of $\frac{1}{2} \delta t \chi_{2}^{2}(1-\alpha)$ with $\alpha$ being the prescribed significance level such as $0.01,0.05$, or 0.1 , we accept the null hypothesis that this wavelet power value is only caused by randomness (i.e. a GWN). Otherwise, the null hypothesis is rejected with a confidence level of $1-\alpha$, and we infer that the wavelet power is significantly larger than those which could be created by randomness. Therefore, it reflects real physical properties of the studied time series.

To demonstrate the significance test for the wavelet power, sine signals with different levels of signal-to-noise ratios, SN, were generated. Each of the signals consists of 2000 points with a sampling frequency of $50 \mathrm{~Hz}$ (i.e. $\delta t=0.02 \mathrm{~s}$ ). The group of signals can be expressed as

$y(t)=A \sin \left(2 \pi f_{x} t\right)+\mathrm{GWN}(0,1)$,

where the peak frequency of the sinusoids $f_{x}$ was always set at $8 \mathrm{~Hz}$ and the term $\operatorname{GWN}(0,1)$ means a GWN with a zero mean and unit variance. $A$ is determined by the prescribed $\mathrm{SN}$ such that $\mathrm{SN}=10 \log _{10} A^{2}$. The wavelet coefficients were calculated using the Fortran code downloaded from TC98's website and further adjusted according to Eq. (5). To concentrate on the significance test, only the wavelet scalogram of 




Fig. 1. Normalized wavelet power of a sinusoid with a GWN $(\mathrm{SN}=3)$ at three instants and the corresponding $5 \%$ significance level.

the central 600 points along the time axis was used (i.e. the 1400 points on both sides were not considered), in order not to complicate the problem with the cone of influence (TC98).

Figure 1 shows three curves of the wavelet powers for frequencies up to $12.5 \mathrm{~Hz}$ at three different times within the 600 point sub-series (i.e. three vertical slices of the wavelet scalogram). The SN of the sine wave was 3, a moderate signalto-noise ratio. It is evident that all the curves have a peak at approximately $8 \mathrm{~Hz}$, and all the peaks are well above the 5\% significance level. Other wavelet power values are below the significance level. From this case we see that the significance test has successfully distinguished the frequency component at $8 \mathrm{~Hz}$ in the sinusoid from other randomly generated fluctuations in the wavelet scalogram.

3.2 The sampling distribution of the wavelet power spectrum of a GWN

Unlike TC98's global wavelet spectrum (Eq. 7), we define the wavelet power spectrum as

$\bar{T}^{2}(a)=\sum_{i=1}^{m}|T(a, i)|^{2}$,

which is simply the sum of the wavelet power over a given time interval at a certain scale $a$. In Eq. (20), $i$ denotes different times in the interval of summation (or integral). For the convenience of discussion, $|T(a, i)|^{2}$ is abbreviated as $|T|_{i}^{2}$ for a certain scale $a$. We would argue that the definition given by Eq. (20) has an advantage over TC98's definition (Eq. 7) in that the summation in Eq. (20) has a direct contribution to the total energy of the studied time series when a further summation (or integral) in scale is taken (e.g. Addison, 2002, p. 29). Therefore, the wavelet spectrum defined through a summation over a time interval seems to be more meaningful and straightforward than that defined through a time averaging. In the following discussion, we proceed with the definition given by Eq. (20). Since the two definitions are only algebraically different by a factor, all results obtained in this section are comparable with TC98's.

First of all, based on the properties of the $\chi_{v}^{2}$ distribution that $E\left[\chi_{v}^{2}\right]=v$ and that $\operatorname{Var}\left[\chi_{v}^{2}\right]=2 v$ (e.g. Jenkins and Watts, 1968 , p. 79), it is obvious that

$E\left[\bar{T}^{2}\right]=\frac{1}{2} \delta t \sigma^{2}(2 m)=m \delta t \sigma^{2}$.

For any $i$ and $j$ that are between 1 and $m$,

$$
\begin{aligned}
& \operatorname{Cov}\left[|T|_{i}^{2},|T|_{j}^{2}\right]=\operatorname{Cov}\left[\iint x(t) x\left(t^{\prime}\right) \psi_{a, i}\left(t^{\prime}\right) \psi_{a, i}^{*}(t) d t d t^{\prime},\right. \\
& \left.\iint x(t) x\left(t^{\prime}\right) \psi_{a, j}\left(t^{\prime}\right) \psi_{a, j}^{*}(t) d t d t^{\prime}\right] \\
& =\int^{(4)} \operatorname{Cov}\left[x(t) x\left(t^{\prime}\right), x\left(t^{\prime \prime}\right) x\left(t^{\prime \prime \prime}\right)\right] \\
& \psi_{a, i}\left(t^{\prime}\right) \psi_{a, i}^{*}(t) \psi_{a, j}\left(t^{\prime \prime \prime}\right) \psi_{a, j}^{*}\left(t^{\prime \prime}\right) d t^{(4)}
\end{aligned}
$$

where $\int^{(4)}[\cdots] d t^{(4)}$ denotes a quadruple integral with respective to the corresponding variables. For $x$ being a GWN, we have

$$
\begin{aligned}
& \operatorname{Cov}\left[x(t) x\left(t^{\prime}\right), x\left(t^{\prime \prime}\right) x\left(t^{\prime \prime \prime}\right)\right]=E\left[x(t) x\left(t^{\prime}\right) x\left(t^{\prime \prime}\right) x\left(t^{\prime \prime \prime}\right)\right] \\
& -E\left[x(t) x\left(t^{\prime}\right)\right] E\left[x\left(t^{\prime \prime}\right) x\left(t^{\prime \prime \prime}\right)\right]
\end{aligned}
$$

and, following Eq. (22), this leads to

$$
\begin{aligned}
& \operatorname{Cov}\left[|T|_{i}^{2},|T|_{j}^{2}\right]=\int^{(4)} E\left[x(t) x\left(t^{\prime}\right) x\left(t^{\prime \prime}\right) x\left(t^{\prime \prime \prime}\right)\right] \\
& \psi_{a, i}\left(t^{\prime}\right) \psi_{a, i}^{*}(t) \psi_{a, j}\left(t^{\prime \prime \prime}\right) \psi_{a, j}^{*}\left(t^{\prime \prime}\right) d t^{(4)}-\delta t^{2} \sigma^{4}
\end{aligned}
$$

(see Appendix $\mathrm{C}$ for more detail).

Based on the derivations in Appendix $\mathrm{C}$, the quadruple integral in Eq. (24) can be decomposed into three terms, namely

$$
\begin{aligned}
& \int^{(4)} E\left[x(t) x\left(t^{\prime}\right) x\left(t^{\prime \prime}\right) x\left(t^{\prime \prime \prime}\right)\right] \psi_{a, i}\left(t^{\prime}\right) \psi_{a, i}^{*}(t) \psi_{a, j} \\
& \left(t^{\prime \prime \prime}\right) \psi_{a, j}^{*}\left(t^{\prime \prime}\right) d t^{(4)}=\delta t^{2} \sigma^{4}\left(I_{1}+I_{2}+I_{3}\right),
\end{aligned}
$$

where

$I_{1}=\left(\int\left|\psi_{a, b}(t)\right|^{2} d t\right)^{2}=1$

(for normalized wavelets in each scale, Eq. (2)),

$$
I_{2}=\left|\int \psi_{a, i}(t) \psi_{a, j}(t) d t\right|^{2},
$$

and

$$
I_{3}=\left|\int \psi_{a, i}(t) \psi_{a, j}^{*}(t) d t\right|^{2} .
$$


Consequently, the covariance of any two wavelet powers at temporal locations $i$ and $j$ is primarily determined by the properties of the associated wavelets.

Substituting in the particular form of the Morlet wavelet, the integral $I_{2}$ and $I_{3}$ can be further reduced (see Appendix C) to yield the following relation

$\operatorname{Cov}\left[|T|_{i}^{2},|T|_{j}^{2}\right]=\delta t^{2} \sigma^{4}\left(1+e^{-\frac{\Delta b^{2}}{2 a^{2}}-2 \omega_{0}^{2}}+e^{-\frac{\Delta b^{2}}{2 a^{2}}}\right)-\delta t^{2} \sigma^{4}$,

with $\Delta b$ denoting the temporal separation between the two time indices $i$ and $j$, i.e. $\Delta b=|i-j| \delta t$. Since the second additive term on the right hand side of Eq. (29) is obviously a small quantity compared with the third term (given $\omega_{0}=6$ ), the second term is hence neglected to yield

$\operatorname{Cov}\left[|T|_{i}^{2},|T|_{j}^{2}\right]=\delta t^{2} \sigma^{4} e^{-\frac{\Delta b^{2}}{2 a^{2}}}$.

Particularly, when $i=j$ (i.e. $\Delta b=0$ ), the covariance becomes variance, and hence

$\operatorname{Var}\left[|T|^{2}\right]=\delta t^{2} \sigma^{4}$.

for any time index $i$. Comparing Eqs. (30) and (31), we deduce that, at a given scale, the covariance of the wavelet power at different times decays exponentially with the increasing temporal separation $\Delta b$. The decay rate is different for different scales. The two equations also constitute the temporal structure of covariance of the wavelet power. This is very similar to the reproducing kernel $K\left(a, t, a^{\prime}, t^{\prime}\right)=\left\langle\psi_{a, t}, \psi_{a^{\prime}, t^{\prime}}\right\rangle$ defined by Mallat (1998, p. 79), where $\langle\cdots\rangle$ means inner product in the $\mathbf{L}^{2}(R)$ space. According to Mallat, $K$ is a measurement of the local redundancy in the wavelet coefficient. In this sense, Eqs. (30) and (31) characterize the local temporal redundancy of a higher-order quantity, the wavelet power, for a GWN. Such redundancy is responsible for the spurious spectral energy of a GWN. Detailed derivation is given in Appendix C.

With the expressions for the variance (Eq. 31) and the covariance (Eq. 30), we are ready to estimate the variance of $\bar{T}^{2}$. Using Eq. (3.2.17) in Jenkins and Watts (1968, p. 73), we have

$$
\operatorname{Var}\left[\bar{T}^{2}\right]=\sum_{i=1}^{m} \operatorname{Var}\left[|T|_{i}^{2}\right]+\sum_{i=1, i \neq j}^{m} \sum_{j=1}^{m} \operatorname{Cov}\left[|T|_{i}^{2},|T|_{j}^{2}\right],
$$

and then

$\operatorname{Var}\left[\bar{T}^{2}\right]=m \delta t^{2} \sigma^{4}+\sum_{i=1, i \neq j}^{m} \sum_{j=1}^{m} \operatorname{Cov}\left[|T|_{i}^{2},|T|_{j}^{2}\right]$.

Based on Eq. (C8),

$\sum_{i=1, i \neq j}^{m} \sum_{j=1}^{m} \operatorname{Cov}\left[|T|_{i}^{2},|T|_{j}^{2}\right]=2 S \delta t^{2} \sigma^{4}$, where $S$ is the sum of the series $\left\{S_{k}=(m-k) e^{-\frac{(k \delta t)^{2}}{2 a^{2}}},(k=\right.$ $1,2, \cdots, m-1)\}$. The value of $S$ can be calculated numerically. More details and properties are given in Appendix C. It therefore follows that

$\operatorname{Var}\left[\bar{T}^{2}\right]=(m+2 S) \delta t^{2} \sigma^{4}$.

In summary, we have obtained the following statistical parameters for the wavelet power spectrum of a GWN:

$E\left[\bar{T}^{2}\right]=m \delta t \sigma^{2}$

and

$\operatorname{Var}\left[\bar{T}^{2}\right]=(m+2 S) \delta t^{2} \sigma^{4}$.

Because of the fact that $\bar{T}^{2}$ is non-negative, we can follow the procedure described in Jenkins and Watts $(1968$, p. 87) to find an approximate distribution for $\bar{T}^{2}$. Specifically, we assume that $\bar{T}^{2} \Rightarrow \gamma \chi_{v}^{2}$ with two parameters $\gamma$ and $v$ to be determined, such that the assumed expected value and variance of $\bar{T}^{2}$ are $\gamma v$ and $2 \gamma^{2} v$, respectively. Matching the actual and deduced expected values and variances, we obtain that

$\gamma=\frac{1}{2} m^{*} \delta t \sigma^{2}$

and

$v=2 \frac{m}{m^{*}}$,

where $m^{*}=1+2 S / m$. Consequently, the sampling distribution of the wavelet spectrum of a GWN is approximately

$\frac{\bar{T}^{2}}{\sigma^{2}} \Rightarrow \frac{1}{2} m^{*} \delta t \chi_{2 m / m^{*}}^{2}$

when the Morlet wavelet is used. Based on this theoretical sampling distribution, a peak in the wavelet power spectrum is considered to be significant (i.e. not caused by pure randomness) with $1-\alpha$ confidence if the normalized peak value, $\bar{T}^{2} / \sigma^{2}$, is larger than the significance level $m^{*} \delta t \chi_{2 m / m^{*}}^{2}(1-\alpha) / 2$, while a peak is considered to be insignificant (i.e. caused by randomness) with $1-\alpha$ confidence if otherwise. It also should be noted from their definitions that the parameters $m$ and $m^{*}$ are both dependent on the scale, so that the significance levels for the wavelet power spectrum is not constant over all scales as that of the wavelet power.

Figure 2 shows a comparison of the significance levels for the normalized wavelet spectrum developed in the present work and those suggested by TC98 for different $\alpha$ values. The two sets of significance levels are in excellent agreement for both cases of $m=40$ and $m=80$, simply supporting the above discussions. Good agreement was also achieved for a variety of other $m$ values, while, for brevity, no more figures are shown here. Figure 3 shows the 5\% significance levels for various $m$ ranging from 5 to 100 (see the figure caption). 



Fig. 2. Significance levels of the normalized wavelet power spectra for (a) $m=40$ and (b) $m=80$; the significance levels, from the top to the bottom, are $1 \%, 5 \%, 10 \%, 15 \%$, and $20 \%$; red solid line: based on Eq. (36); blue circle: according to TC98.

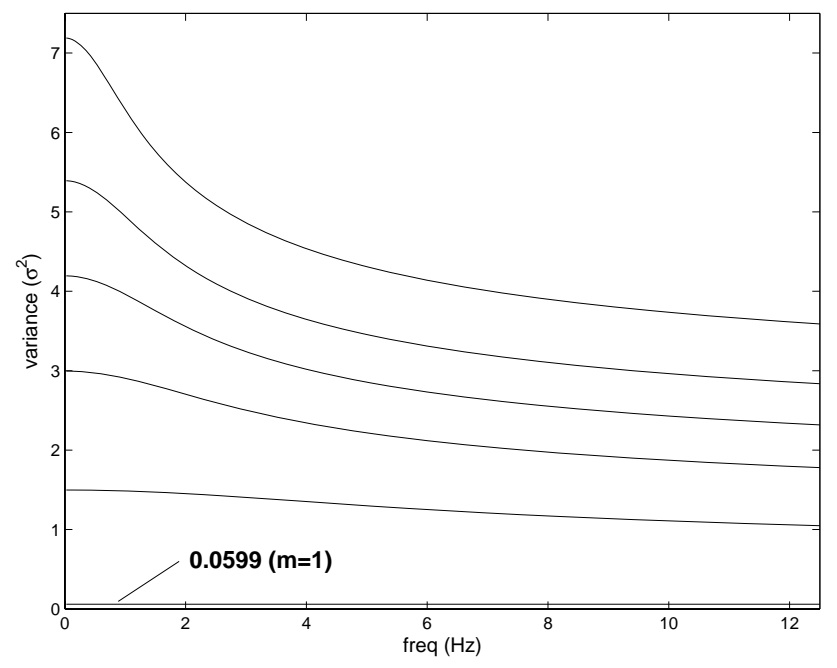

Fig. 3. 5\% significance levels of the normalized wavelet power spectra for, from the top to the bottom, $m=120, m=90, m=70$, $m=50, m=25$, and $m=1$ all based on Eq. (36).

Besides an obvious property that the significance level increases as increasing $m$ (the number of the points covered by the integral or the summation), it also is evident that the significance level approaches a level line as $m$ decreases. When $m$ becomes 1 , the significance level of the wavelet spectrum should ideally become that of the wavelet power. As a matter of fact, the 5\% significance level for $m=1$ was estimated to be 0.0599 using Eq. (36), in excellent agreement again with the theoretical value, 0.06, given by Eq. (18) and shown in Fig. 1.

For the test signal given by Eq. (19) with a SN of 3, three wavelet power spectra were obtained by integrating

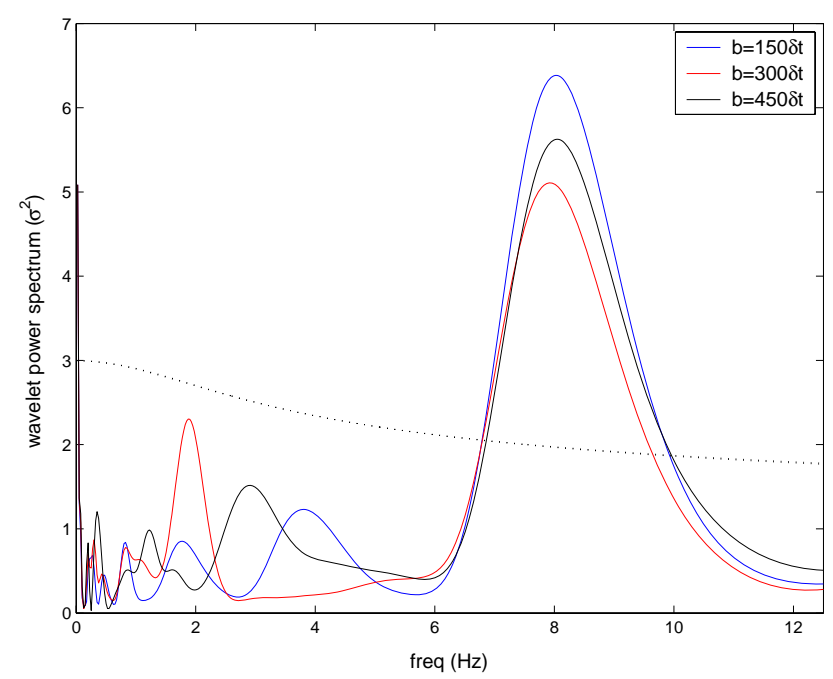

Fig. 4. Normalized wavelet power spectra of a sinusoid with a GWN ( $\mathrm{SN}=3$ ) integrated over $m=50$ points starting from three instants: $b=150 \delta t, b=300 \delta t$, and $b=450 \delta t$; dotted line: the corresponding $5 \%$ significance level.

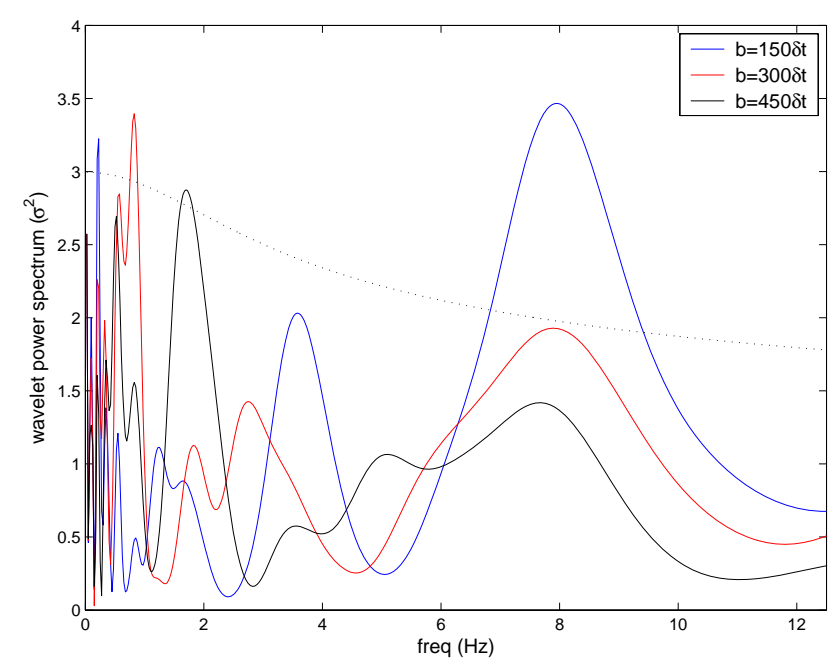

Fig. 5. Normalized wavelet power spectra of a sinusoid with a GWN ( $\mathrm{SN}=-3)$ integrated over $m=50$ points starting from three instants: $b=150 \delta t, b=300 \delta t$, and $b=450 \delta t$; dotted line: the corresponding $5 \%$ significance level.

the wavelet power over $m=50$ points at three starting locations in the 600-point time interval: $b=150 \delta t, b=300 \delta t$, and $b=450 \delta t$, and were further normalized by their respective variances, $\sigma^{2}$, in the time domain (Fig. 4). Because of the high signal-to-noise ratio, the peaks at $8 \mathrm{~Hz}$ in all the three spectra are well above the 5\% significance level. It therefore is inferred that these three peaks are all significant, or not due to randomness. In comparison, a smaller peak is visible at $2 \mathrm{~Hz}$ in the wavelet spectrum for the case of $b=300 \delta t$. This peak is considered to be fortuitous (created by randomness) because it is still below the local 5\% significance level. 


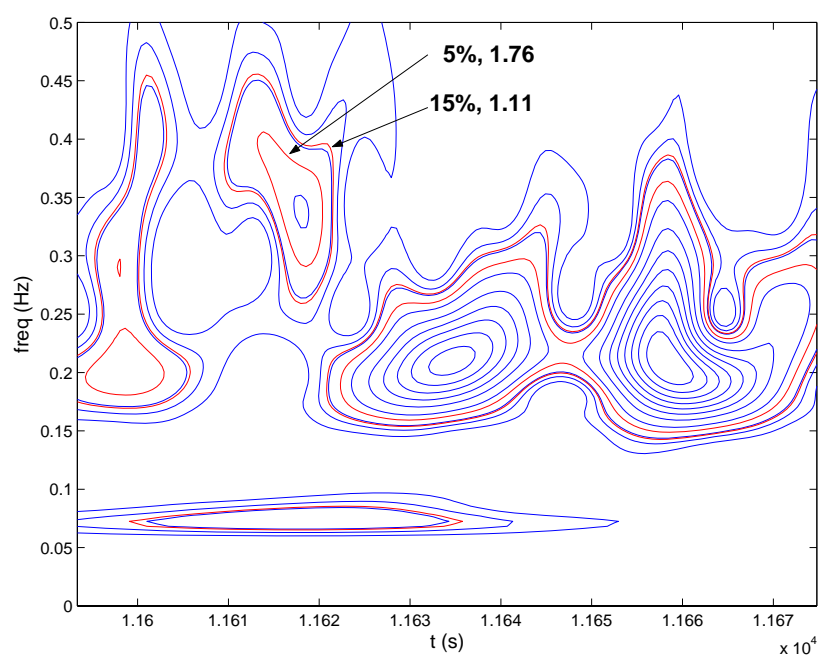

Fig. 6. Normalized wavelet scalogram of an actual time series of wave elevation: 12 contours (blue) for the wavelet scalogram and two contours (red) for the $5 \%$ and $15 \%$ significance levels.

When the $\mathrm{SN}$ is reduced to -3 , the wavelet spectra obtained by integrating over the same three periods are shown in Fig. 5. Owing to the overwhelming noise content, only the wavelet spectrum for the case of $b=150 \delta t$ has a significant peak at $8 \mathrm{~Hz}$. The peaks at the same frequency in the other two spectra were completely contaminated by the noise.

\section{Significance tests on actual observations}

We go beyond the simulated signals in this section. When actual data are to be analysed, the theoretically meaningful 5\% probability may be too stringent. This is due to the fact that the signal-to-noise ratio in naturally observed data is often low if not negative. In such cases, the threshold significance level can empirically be relaxed to $15 \%$ or even higher, depending on the particular problems.

An actual data set is studied here as an example of application in geophysics. The data set consists of a time series of wave elevation recorded from the 3-m discus buoy 45011 of the NOAA National Data Buoy Center (NDBC), which was deployed during the autumn of 1997 in nearshore eastern Lake Michigan of the United States. Specifically, the buoy was at $10 \mathrm{~m}$ water depth at $43.02^{\circ} \mathrm{N}, 86.27^{\circ} \mathrm{W}$, about $1.5 \mathrm{~km}$ southwest of Grand Haven, Michigan. The sampling frequency $F_{S}$ was approximately $1.70667 \mathrm{~Hz}$. The detailed structure of the buoy and the sampling processes are the same as described by Ge and Liu (2007).

Figure 6 shows the normalized wavelet scalogram of the wave elevation during the same period as stage I and stage II in Ge and Liu (2007). There are 140 data points in this period, which covers about $80 \mathrm{~s}$. In Ge and Liu (2007), the first half $(40 \mathrm{~s})$ of the period is referred to as stage I and the second stage II. From the wavelet scalogram, we observed

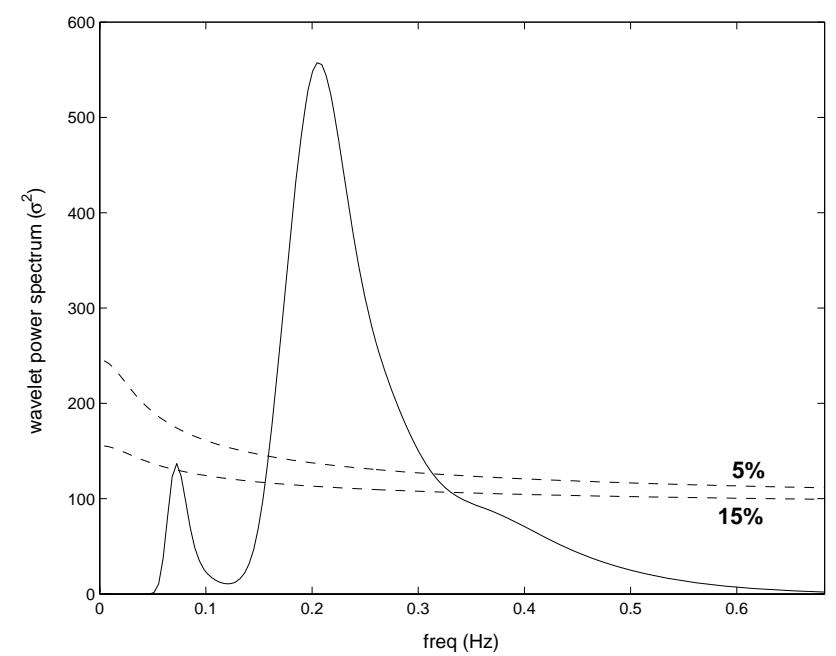

Fig. 7. Normalized wavelet power spectrum of the same wave elevation time series as in Fig. 6 integrated over $m=140$ points and the $5 \%$ and $15 \%$ significance levels.

different patterns for the two stages. The spectral peak at around $0.2 \mathrm{~Hz}$ was considerably enhanced during stage II, while another peak at around $0.08 \mathrm{~Hz}$ disappeared in this stage. Against the 5\% and $15 \%$ significance levels, it is clear that the major patterns we have observed are all significant. Particularly, it is noted that the broad peak at $0.08 \mathrm{~Hz}$, which extended across the entire stage I, should be insignificant if 95\% confidence is required. Obviously, a higher significance level, such as $15 \%$, was adopted by Ge and Liu (2007) in order not to overlook any physically meaningful patterns (e.g. Fig. 5 of $\mathrm{Ge}$ and Liu, 2007). It also is noted that, without significance tests, one would misinterpret the peak at $0.25 \mathrm{~Hz}$ and approximately $11665 \mathrm{~s}$, as well as the large bump at around $0.3 \mathrm{~Hz}$ from 11600 to $11614 \mathrm{~s}$, which are most likely to be fortuitous.

The normalized wavelet power spectrum of the wave elevation is shown in Fig. 7 along with the levels for 5\% and $15 \%$ of significance, respectively. For this particular case, the integral was over the whole 140-point range, so that $m=140$. The peak at approximately $0.2 \mathrm{~Hz}$ is very conspicuous, while another peak at about $0.08 \mathrm{~Hz}$ appears to be relatively small and almost below the both significance levels. We hence infer from Fig. 7 that the peak at $0.2 \mathrm{~Hz}$ is statistically significant with 95\% confidence throughout the entire 140-point period, whereas the peak at $0.08 \mathrm{~Hz}$ is not even significant with $85 \%$ confidence. On the other hand, it should be noted that these inferences are for the case of $m=140$. The insignificance of the peak at $0.08 \mathrm{~Hz}$ is due to the fact that the peak is primarily confined in stage I. This means that, when the wavelet spectrum is only estimated for stage $\mathrm{I}(m=70)$, the peak at $0.08 \mathrm{~Hz}$ should become significant (Fig. 6). 


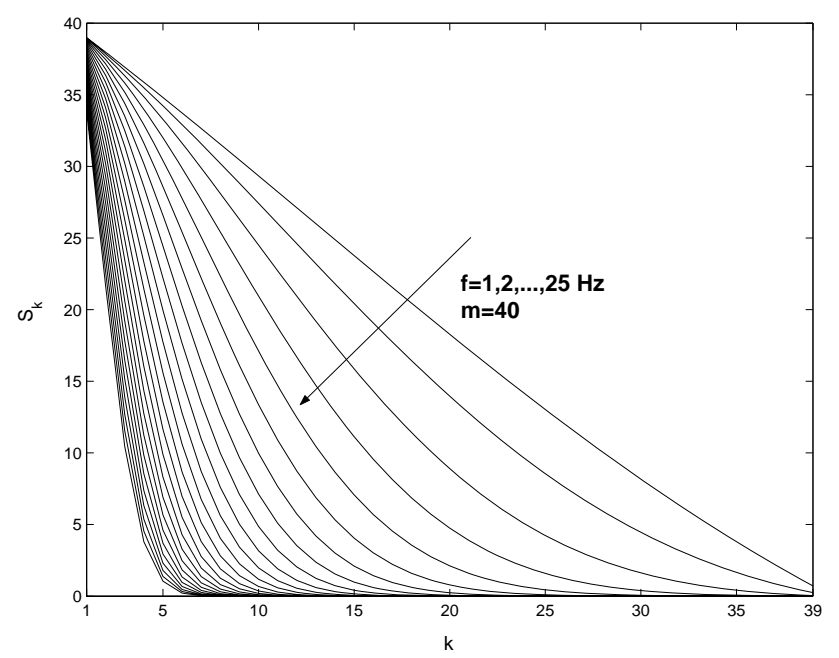

Fig. 8. Property of the series $\left\{S_{k}, k=1,2, \cdots, m-1\right\}$ and its dependence on the frequency (scale) for the case of $m=40$; the curves from the top to the bottom are for the frequencies of $1,2, \ldots, 25 \mathrm{~Hz}$.

\section{Further discussion}

In the previous sections we have developed significance tests for the wavelet power and the wavelet power spectrum. The tests are against a particular background noise, a GWN. One may further be concerned with the confidence interval (CI) of the wavelet-based quantities, since, for ordinary statistics, the CI can be easily obtained through similar derivations as one does for significance tests. This essentially benefits from the stationarity of the studied time series, whose statistics can be completely determined by its lowest moments (e.g. mean and variance). This, however, is not true for nonstationary time series. Over a short period, the behaviour of a non-stationary time series cannot be characterized in a statistical sense, leaving its CI meaningless. As Qiu and Er (1995) showed, the variance (and hence other higher-order moments) of the wavelet power of a noise-contaminated signal depends on both the noise level and the signal itself. But the signal part, which is often non-stationary, is what we do not know. The expression for the CI should not be as simple as, for example, Eq. (20) of TC98.

It also is clear that the wavelet family that is used for analysis is critical to the significance tests of, at least, the wavelet power and power spectrum. The role of the wavelet family is typically realized through the series $S_{k}$ and hence $m^{*}$ in Eq. (36), and shown in Fig. 8. The above evidence is obviously contrary to TC 98 's statement that the significance tests "should not depend upon the wavelet function or upon the actual distribution of the time series, other than the assumption of a background spectrum."

Finally, it is important to point out that other factors, for example, the edge effect (TC98) and the aliasing problem (Jordan, Miksad, and Powers, 1997), also need be considered for proper significance tests. One should make sure that the wavelet-based results are really free from such contaminations before proceeding to significance tests. Otherwise, no statistical test is promised to be effective. These issues are, however, beyond the scope of the present paper.

\section{Conclusions}

Significance tests cannot be omitted when statistical inferences are to be drawn from a single realization (sample) of a population. This certainly is true when one attempts to interpret the wavelet-based statistical results of the studied nonstationary process. The significance test answers the question as to what part of the results may have been created by pure randomness and what part represents true physics. TC98 accurately estimated the significance levels for the wavelet power and power spectrum against a background white/red noise. However, the significance levels were obtained empirically through Monte Carlo simulations. Although numerically accurate, the Monte Carlo simulations employed in TC98 failed to disclose many statistical properties that are interesting to users of the wavelet analysis.

Based on a rigorous statistical analysis, the present work estimated the sampling distributions for the wavelet power and the wavelet power spectrum of a GWN, through which the significance tests for the two fundamental quantities of the wavelet analysis were established. It was found that the sampling distribution of the wavelet power of a GWN process is identical to TC98's form adjusted by $\delta t$. The significance levels of the wavelet power spectrum of a GWN are numerically in excellent agreement with TC98's results, while we believe that the expressions given in the present work are more convenient and reflect more statistical properties. We thus recommend that TC98's expressions should be replaced by their corresponding ones developed here. It also was demonstrated that the sampling distributions, and hence the significance tests, are highly related to the particular wavelet family one chooses to use. For other wavelet functions than the Morlet wavelet, neither may the sampling distribution of the wavelet power of a GWN be a $\chi_{2}^{2}$ distribution (see Eq. 16), nor will the sampling distribution of the wavelet spectrum take the same form simply because $m^{*}$ in Eq. (36) varies with different wavelet families. Another important property concerns the structure of covariance of the wavelet power of a GWN at different temporal locations, as described by Eqs. (30) and (31). This property bears resemblance to the reproducing kernel, which describes the redundancy in the wavelet coefficient. These findings disagree with TC98's statements that the significance tests should be independent of the wavelet family and the actual distribution of the studied time series.

The significance tests developed in the present work were demonstrated on both simulated and naturally observed time series. The simulated noisy sinusoids illustrated the fact that pure noise may create false peaks everywhere. For the actual 
wave elevation time series observed from a buoy deployed on Lake Michigan, the significance tests clearly indicated different significance levels for different peaks, and helped to avoid misinterpretation of two fortuitous peaks in the wavelet scalogram. They are all examples for the necessity and effectiveness of the significance tests proposed in the present work.

The significance tests for other fundamental quantities in the wavelet analysis, such as the wavelet cross-spectrum, coherency, and the higher-order moments, will be discussed in subsequent works.

\section{Appendix A}

\section{Adjustment for the results in TC98}

There are more than one approaches to prove that $|T(a, b)|^{2}=\delta t\left|W_{n}(a)\right|^{2} \quad$ (Eq. 5). For example, using TC98's Eq. (8), $\psi\left[\frac{\left(n^{\prime}-n\right) \delta t}{a}\right]=\frac{\sqrt{\delta t}}{\sqrt{a}} \psi_{0}\left[\frac{\left(n^{\prime}-n\right) \delta t}{a}\right]$, we have $\psi^{*}\left[\frac{\left(n^{\prime}-n\right) \delta t}{a}\right]=\frac{\sqrt{\delta t}}{\sqrt{a}} \psi_{0}^{*}\left[\frac{\left(n^{\prime}-n\right) \delta t}{a}\right]$, where $\psi_{0}$ denotes the mother wavelet as in TC98's notation. Hence, TC98's definition for the wavelet coefficient, Eq. (4), becomes

$$
W_{n}(a)=\sum_{n^{\prime}=0}^{N-1} x_{n^{\prime}} \frac{\sqrt{\delta t}}{\sqrt{a}} \psi_{0}^{*}\left[\frac{\left(n^{\prime}-n\right) \delta t}{a}\right] \text {. }
$$

On the other hand, the discrete form of Eq. (3), the standard definition for the wavelet coefficients in the literature, is

$T_{n}(a)=\sum_{n^{\prime}=0}^{N-1} x_{n^{\prime}} \frac{\delta t}{\sqrt{a}} \psi_{0}^{*}\left[\frac{\left(n^{\prime}-n\right) \delta t}{a}\right]$.

A comparison of the two discrete forms, Eqs. (4) and (A2), immediately leads to

$T_{n}(a)=\sqrt{\delta t} W_{n}(a)$,

and hence Eq. (5). This means that the wavelet coefficients and the higher-order quantities defined in TC98 or calculated using their source code should be adjusted accordingly to reconcile with the results with standard definitions, as those in the present work. The adjustment relation, Eq. (5), was also verified numerically by comparing the results given by TC98's Fortran code and their corresponding analytical results for a set of test signals.

\section{Appendix B}

\section{Variance of the real and imaginary parts of the wavelet coefficient of a GWN}

Equation (12) states that $\operatorname{Var}[\operatorname{Re}[T]]=$ $\iint E\left[x(t) x\left(t^{\prime}\right)\right] \operatorname{Re}\left[\psi_{a, b}^{*}(t)\right] \operatorname{Re}\left[\psi_{a, b}^{*}\left(t^{\prime}\right)\right] d t d t^{\prime}$. This relation can be further simplified making use of the property of the GWN $x(t)$. The discrete form for Eq. (12) is

$\operatorname{Var}[\operatorname{Re}[T]]=$

$\sum_{i} \sum_{j} E\left[x\left(t_{i}\right) x\left(t_{j}\right)\right] \operatorname{Re}\left[\psi_{a, b}^{*}\left(t_{i}\right)\right] \operatorname{Re}\left[\psi_{a, b}^{*}\left(t_{j}\right)\right] \delta t^{2}$,

and equivalently,

$\operatorname{Var}[\operatorname{Re}[T]]=$

$\delta t^{2} \sum_{i} E\left[x\left(t_{i}\right) x\left(t_{i}\right)\right] \operatorname{Re}\left[\psi_{a, b}^{*}\left(t_{i}\right)\right] \operatorname{Re}\left[\psi_{a, b}^{*}\left(t_{i}\right)\right]$.

Therefore

$\operatorname{Var}[\operatorname{Re}[T]]=\delta t \sigma^{2} \sum_{i} \operatorname{Re}^{2}\left[\psi_{a, b}^{*}\left(t_{i}\right)\right] \delta t$.

This obviously is the discrete form for Eq. (13). The variance of the imaginary part of the wavelet coefficient can be derived similarly.

\section{Appendix C}

\section{More detailed derivations for the wavelet power spectrum of a GWN}

Since

$\operatorname{Cov}\left[x(t) x\left(t^{\prime}\right), x\left(t^{\prime \prime}\right) x\left(t^{\prime \prime \prime}\right)\right]=E\left[\left(x(t) x\left(t^{\prime}\right)-\right.\right.$

$\left.\left.E\left[x(t) x\left(t^{\prime}\right)\right]\right)\left(x\left(t^{\prime \prime}\right) x\left(t^{\prime \prime \prime}\right)-E\left[x\left(t^{\prime \prime}\right) x\left(t^{\prime \prime \prime}\right)\right]\right)\right]$,

this further results in

$\operatorname{Cov}\left[x(t) x\left(t^{\prime}\right), x\left(t^{\prime \prime}\right) x\left(t^{\prime \prime \prime}\right)\right]=E\left[x(t) x\left(t^{\prime}\right) x\left(t^{\prime \prime}\right) x\left(t^{\prime \prime \prime}\right)\right]$

$-E\left[x(t) x\left(t^{\prime}\right)\right] E\left[x\left(t^{\prime \prime}\right) x\left(t^{\prime \prime \prime}\right)\right]$

(Eq. 23) after some algebra. The quadruple integral of the term $E\left[x(t) x\left(t^{\prime}\right)\right] E\left[x\left(t^{\prime \prime}\right) x\left(t^{\prime \prime \prime}\right)\right]$ is

$\int^{(4)} E\left[x(t) x\left(t^{\prime}\right)\right] E\left[x\left(t^{\prime \prime}\right) x\left(t^{\prime \prime \prime}\right)\right] \psi_{a, i}\left(t^{\prime}\right) \psi_{a, i}^{*}(t) \psi_{a, j}$
$\left(t^{\prime \prime \prime}\right) \psi_{a, j}^{*}\left(t^{\prime \prime}\right) d t^{(4)}=\delta t^{2} \sigma^{4}$

based on the properties of $x(t)$ following a similar procedure as in Appendix B. Equation (C1) explains the last term on the right hand side of Eq. (24).

According to Jenkins and Watts (1968, p. 206),

$$
\begin{aligned}
& E\left[x(t) x\left(t^{\prime}\right) x\left(t^{\prime \prime}\right) x\left(t^{\prime \prime \prime}\right)\right]=\sigma^{4}\left[\delta\left(t^{\prime}-t\right) \delta\left(t^{\prime \prime \prime}-t^{\prime \prime}\right)\right. \\
& \left.+\delta\left(t^{\prime \prime}-t\right) \delta\left(t^{\prime \prime \prime}-t^{\prime}\right)+\delta\left(t^{\prime \prime \prime}-t\right) \delta\left(t^{\prime \prime}-t^{\prime}\right)\right]
\end{aligned}
$$

for $x(t)$ being a GWN and hence the $\kappa_{4}$ term is zero (Jenkins and Watts, 1968, p. 175). Therefore,

$\int^{(4)} E\left[x(t) x\left(t^{\prime}\right) x\left(t^{\prime \prime}\right) x\left(t^{\prime \prime \prime}\right)\right] \psi_{a, i}\left(t^{\prime}\right) \psi_{a, i}^{*}(t) \psi_{a, j}\left(t^{\prime \prime \prime}\right) \psi_{a, j}^{*}\left(t^{\prime \prime}\right) d t^{(4)}$

$$
\begin{aligned}
& =\delta t^{2} \sigma^{4} \int^{(4)} \frac{1}{\delta t^{2}}\left[\delta\left(t^{\prime}-t\right) \delta\left(t^{\prime \prime \prime}-t^{\prime \prime}\right)\right. \\
& \left.+\delta\left(t^{\prime \prime}-t\right) \delta\left(t^{\prime \prime \prime}-t^{\prime}\right)+\delta\left(t^{\prime \prime \prime}-t\right) \delta\left(t^{\prime \prime}-t^{\prime}\right)\right]
\end{aligned}
$$


$\psi_{a, i}\left(t^{\prime}\right) \psi_{a, i}^{*}(t) \psi_{a, j}\left(t^{\prime \prime \prime}\right) \psi_{a, j}^{*}\left(t^{\prime \prime}\right) d t^{(4)}$.

Now the three terms in the integrand are evaluated separately.

$I_{1}=\frac{1}{\delta t^{2}} \int^{(4)} \delta\left(t^{\prime}-t\right) \delta\left(t^{\prime \prime \prime}-t^{\prime \prime}\right) \psi_{a, i}\left(t^{\prime}\right) \psi_{a, i}^{*}(t) \psi_{a, j}$

$\left(t^{\prime \prime \prime}\right) \psi_{a, j}^{*}\left(t^{\prime \prime}\right) d t^{(4)}=\iint\left|\psi_{a, i}(t)\right|^{2}\left|\psi_{a, j}\left(t^{\prime \prime}\right)\right|^{2} d t d t^{\prime \prime}$

$=\left(\int\left|\psi_{a, b}(t)\right|^{2} d t\right)^{2}=1$

for such normalized wavelets as in Eq. (2). Similarly,

$$
\begin{aligned}
& I_{2}=\frac{1}{\delta t^{2}} \int^{(4)} \delta\left(t^{\prime \prime}-t\right) \delta\left(t^{\prime \prime \prime}-t^{\prime}\right) \psi_{a, i}\left(t^{\prime}\right) \psi_{a, i}^{*}(t) \psi_{a, j} \\
& \left(t^{\prime \prime \prime}\right) \psi_{a, j}^{*}\left(t^{\prime \prime}\right) d t^{(4)}=\int \psi_{a, i}^{*}(t) \psi_{a, j}^{*}(t) d t \int \psi_{a, i}(t) \psi_{a, j}(t) d t \\
& =\left|\int \psi_{a, i}(t) \psi_{a, j}(t) d t\right|^{2},
\end{aligned}
$$

and

$$
\begin{aligned}
& I_{3}=\frac{1}{\delta t^{2}} \int^{(4)} \delta\left(t^{\prime \prime \prime}-t\right) \delta\left(t^{\prime \prime}-t^{\prime}\right) \psi_{a, i}\left(t^{\prime}\right) \psi_{a, i}^{*}(t) \psi_{a, j} \\
& \left(t^{\prime \prime \prime}\right) \psi_{a, j}^{*}\left(t^{\prime \prime}\right) d t^{(4)}=\iint \psi_{a, i}\left(t^{\prime}\right) \psi_{a, i}^{*}(t) \psi_{a, j}(t) \psi_{a, j}^{*}\left(t^{\prime}\right) d t d t^{\prime} \\
& =\int \psi_{a, i}(t) \psi_{a, j}^{*}(t) d t \int \psi_{a, i}^{*}(t) \psi_{a, j}(t) d t \\
& =\left|\int \psi_{a, i}(t) \psi_{a, j}^{*}(t) d t\right|^{2} .
\end{aligned}
$$

Substituting the Morlet wavelet into the above expressions, we further simplified $I_{2}$ and $I_{3}$ to be

$$
I_{2}=e^{-\frac{\Delta b^{2}}{2 a^{2}}-2 \omega_{0}^{2}}
$$

and

$$
I_{3}=e^{-\frac{\Delta b^{2}}{2 a^{2}}}
$$

where $\Delta b=|i-j| \delta t$ for the separation between the time indices $i$ and $j$. Consequently, we obtained Eq. (29).

Next step here is to simplify the summation $\sum_{i=1, i \neq j}^{m} \sum_{j=1}^{m} \operatorname{Cov}\left[|T|_{i}^{2},|T|_{j}^{2}\right]$ as in Eq. (32). By enumerating all possible separations for $i$ and $j$, we have

$$
\sum_{i=1, i \neq j}^{m} \sum_{j=1}^{m} \operatorname{Cov}\left[|T|_{i}^{2},|T|_{j}^{2}\right]
$$

$$
\begin{aligned}
& =2\left\{\left.\operatorname{Cov}\left[|T|_{i}^{2},|T|_{j}^{2}\right]\right|_{i<j, \Delta b=\delta t}+\left.\operatorname{Cov}\left[|T|_{i}^{2},|T|_{j}^{2}\right]\right|_{i<j, \Delta b=2 \delta t}\right. \\
& \left.+\cdots+\left.\operatorname{Cov}\left[|T|_{i}^{2},|T|_{j}^{2}\right]\right|_{i<j, \Delta b=(m-1) \delta t}\right\}
\end{aligned}
$$

$$
\begin{aligned}
& =2\left\{(m-1) e^{-\frac{\delta t^{2}}{2 a^{2}}}+(m-2) e^{-\frac{(2 \delta t)^{2}}{2 a^{2}}}+\cdots\right. \\
& \left.+1 \cdot e^{-\frac{[(m-1) \delta t]^{2}}{2 a^{2}}}\right\} \delta t^{2} \sigma^{4} \\
& =2 \delta t^{2} \sigma^{4} \sum_{k=1}^{m-1}(m-k) e^{-\frac{(k \delta t)^{2}}{2 a^{2}}},
\end{aligned}
$$

and finally

$$
\sum_{i=1, i \neq j}^{m} \sum_{j=1}^{m} \operatorname{Cov}\left[|T|_{i}^{2},|T|_{j}^{2}\right]=2 \delta t^{2} \sigma^{4} S,
$$

where

$S=\sum_{k=1}^{m-1} S_{k}=\sum_{k=1}^{m-1}(m-k) e^{-\frac{(k \delta t)^{2}}{2 a^{2}}}$.

It is important to note here that the $S_{k}$ series in Eq. (C9) is not convergent but dependent on the parameter $m$ and the particular wavelet function. The best way to find the value of $S$ seems to be to write a programme and do the summation in Eq. (C9) numerically. Moreover, each term in the $S_{k}$ series has an exponentially decaying trend whose decay rate is determined by the temporal separation of the two locations, $\Delta b$ or $k \delta t$. It is also noted that the $S_{k}$ series is scale-dependent, and, more rigorously, the series should be denoted as $S_{k}(a)$ or $S_{k}(f)$. Figure 8 shows the distribution of the series $\left\{S_{k}(f), k=1,2, \cdots, m-1\right\}$ for the case of $m=40$. For different frequencies (scales), the series are different. Hence the 25 curves in Fig. 8 reveal the scale-dependence of $S_{k}(f)$. It is now evident that at very low frequencies such as $1 \mathrm{~Hz} S_{k}$ does not decay fast with increasing temporal separation. In this case no convergence could be expected throughout the 39 terms. This is a natural result of the large temporal support of the wavelet at large scales. In contrast, at a much higher frequency such as $25 \mathrm{~Hz}, S_{k}$ decays to near zero at $k=7$, implying fast decorrelation of the wavelets at small scales.

After collecting involved terms, we obtain Eq. (33): $\operatorname{Var}\left[\bar{T}^{2}\right]=(m+2 S) \delta t^{2} \sigma^{4}$.

Acknowledgements. The author wishes to thank the Research Associateship Programs of the National Research Council for its financial support. Walter Frick of USEPA and P. C. Liu of NOAA are also thanked for their support and helpful discussions. This paper has been reviewed in accordance with the U.S. Environmental Protection Agency's peer and administrative review policies and approved for publication. Mention of trade names or commercial products does not constitute endorsement or recommendation for use.

Topical Editor S. Gulev thanks two anonymous referees for their help in evaluating this paper.

\section{References}

Addison, P. S: The illustrated wavelet transform handbook: Introductory theory and applications in science, engineering, medicine and finance, IOP Publishing Ltd., Bristol, UK, 2002. 
Foufoula-Georgiou, E. and Kumar, P. (Eds): Wavelets in Geophysics, Academic, San Diego, 1994.

Ge, Z. and Liu, P. C.: A time-localized response of wave growth process under turbulent winds, Ann. Geophys., 25, 1253-1262, 2007,

http://www.ann-geophys.net/25/1253/2007/.

Goupilland, P., Grossman, A., and Morlet, J.: Cycle-Octare and related transforms in seismic signal analysis, Geoexploration, 23, 85-105, 1984.

Jenkins, G. M. and Watts, D. G.: Spectral analysis and its applications, Holden-Day, San Francisco, 1968.
Jordan, D., Miksad, R. W., and Powers, E. J.: Implementation of the continuous wavelet transform for digital time series analysis, Rev. Sci. Instrum., 68, 1484-1494, 1997.

Mallat, S.: A wavelet tour of signal processing, Academic Press, San Diego, CA, 1998.

Qiu, L. and Er, M. H.: Wavelet spectrogram of noisy signals, Int. J. Electronics., 79, 665-677, 1995.

Torrence, C. and Compo, G. P.: A practical guide to wavelet analysis, Bull. Am. Meteorol. Soc., 79, 61-78, 1998. 